\section{A conserved ncRNA-binding protein recruits silencing factors to heterochromatin through an RNAi-independent mechanism}

\author{
Diana B. Marina, ${ }^{1}$ Smita Shankar, ${ }^{1}$ \\ Prashanthi Natarajan, Kenneth J. Finn, \\ and Hiten D. Madhani ${ }^{2}$ \\ Department of Biochemistry and Biophysics, University of \\ California at San Francisco, San Francisco, California 94158, USA
}

Long noncoding RNAs (IncRNAs) can trigger repressive chromatin, but how they recruit silencing factors remains unclear. In Schizosaccharomyces pombe, heterochromatin assembly on transcribed noncoding pericentromeric repeats requires both RNAi and RNAi-independent mechanisms. In Saccharomyces cerevisiae, which lacks a repressive chromatin mark (H3K9me [methylated Lys9 on histone $\mathrm{H} 3]$ ), unstable ncRNAs are recognized by the RNA-binding protein Nrd1. We show that the $S$. pombe ortholog Seb1 is associated with pericentromeric lncRNAs. Individual mutation of $\mathrm{dcr}^{+}$(Dicer) or seb1 ${ }^{+}$results in equivalent partial reductions of pericentromeric $\mathrm{H} 3 \mathrm{~K} 9 \mathrm{me}$ levels, but a double mutation eliminates this mark. Seb1 functions independently of RNAi by recruiting the NuRD (nucleosome remodeling and deacetylase)-related chromatin-modifying complex SHREC (Snf2-HDAC [histone deacetylase] repressor complex).

Supplemental material is available for this article.

Received November 1, 2012; revised version accepted August 7, 2013.

A major unsolved question in chromatin biology is how long intergenic noncoding RNAs (lincRNAs) trigger the formation of repressed chromatin. A large number of mammalian lincRNAs have been identified by systematic studies (Guttman et al. 2009). Many of these ncRNAs associate with chromatin-modifying complexes (Khalil et al. 2009). Multiple models have been proposed for how these ncRNAs are recognized and recruit chromatinmodifying factors, but little is understood mechanistically (Guttman and Rinn 2012).

In Schizosaccharomyces pombe, pericentromeric heterochromatin assembly is promoted by transcription of the $d g$ and $d h$ repeat sequences by RNA polymerase II (Pol II) (Djupedal et al. 2005; Kato et al. 2005). The corresponding long ncRNAs (lncRNAs) are converted into dsRNAs and processed into siRNAs by the combined action of

[Keywords: Seb1; Nrd1; RNAi; heterochromatin; SHREC; silencing]

${ }^{1}$ These authors contributed equally to this work.

${ }^{2}$ Corresponding author

E-mail hitenmadhani@gmail.com

Article is online at http://www.genesdev.org/cgi/doi/10.1101/gad.226019.113.
RNA-directed RNA polymerase complex (RDRC) and Dicer (Dcr1) (Verdel et al. 2009; Lejeune and Allshire 2011). siRNAs produced by Dicer are bound by Argonaute (Ago1), a component of the RNA-induced transcriptional silencing (RITS) complex, and together they promote both degradation of pericentromeric ncRNAs and transcriptional silencing via repressive histone methylation (Verdel et al. 2004). These complexes in turn recruit the Clr4 methyltransferase complex (CLRC), which methylates Lys9 on histone H3 (H3K9me) (Nakayama et al. 2001; Zhang et al. 2008). The methyl mark serves as a binding platform for the repressive HP1 proteins Swi6 and Chp2 (Thon and Verhein-Hansen 2000; Bannister et al. 2001; Fischer et al. 2009). Both proteins promote the recruitment of SHREC [Snf2-HDAC [histone deacetylase] repressor complex) to pericentromeric heterochromatin (Sugiyama et al. 2007; Sadaie et al. 2008). Moreover, Chp2 has been found to associate with SHREC to form the SHREC2 complex (SHREC complex associated with Chp2) (Motamedi et al. 2008). The core of SHREC consists of silencing factors $\mathrm{Clr} 1$ and $\mathrm{Clr} 2$, the HDAC Clr3, and the putative chromatin-remodeling enzyme Mitl (Sugiyama et al. 2007). SHREC and SHREC2 resemble the mammalian nucleosome remodeling and deacetylase (NuRD) complex (Sugiyama et al. 2007; Motamedi et al. 2008). Previous studies revealed that deletion of $c l r 3^{+}$reduces the levels of pericentromeric H3K9me2 in cells lacking RNAi (Yamada et al. 2005; Reyes-Turcu et al. 2011), indicating that Clr3/SHREC can act independently of RNAi in pericentromeric heterochromatin assembly.

In addition to processing by the RNAi machinery, the pericentromeric lncRNAs are also recognized by several RNA quality control factors such as Mlo3 and Cid14 (Reyes-Turcu et al. 2011). Interestingly, mutations in these factors have been shown to suppress the silencing defect of RNAi mutants at pericentromeric repeats (Reyes-Turcu et al. 2011). This led to the suggestion that the ncRNAs might also act in the RNAi-independent pathway of heterochromatin assembly (Reyes-Turcu et al. 2011). Here we identify a conserved ncRNA-binding protein, Seb1/Nrd1, which binds pericentromeric ncRNAs and is required for H3K9me in cells deficient for RNAi. We demonstrate that this ncRNA-binding protein functions in the RNAiindependent pathway by recruiting the activities of SHREC.

\section{Results and Discussion}

To investigate how ncRNAs lead to the formation of repressed chromatin, we identified a candidate ncRNA recognition factor in $S$. pombe based on prior studies of ncRNAs in Saccharomyces cerevisiae. There, a class of unstable ncRNAs called cryptic unstable transcripts (CUTs) is recognized by an RNA-binding protein called Nrdl (Arigo et al. 2006; Thiebaut et al. 2006). Nrdl globally associates with Pol II via a C-terminal domain-interacting domain (CID) and recognizes a specific RNA oligonucleotide through its RNA recognition motif (RRM) domain (Steinmetz and Brow 1996, 1998; Conrad et al. 2000; Carroll et al. 2004; Meinhart and Cramer 2004; Vasiljeva et al. 2008a). Nrdl also plays a role in recognizing precursors to stable ncRNAs such as nucleolar RNAs (snoRNAs) and small nuclear RNAs (snRNAs) to promote their 3' end formation (Steinmetz et al. 2001; Kim et al. 
2006). Although the $S$. cerevisiae lineage lost both H3K9me and RNAi during its evolution, we hypothesized that the role of Nrd1 in ncRNA recognition might be conserved. To test this, we tagged the $S$. pombe Nrd1 ortholog Seb1 (Mitsuzawa et al. 2003) and used cross-linking and RNA immunoprecipitation (RIP) to assess its association with $d g$ and $d h$ ncRNAs as well as the snoRNA $s n R 30$. Seb1 displays a strong association with these ncRNAs but not with the $a c t 1^{+}$RNA (Fig. 1A). As expected, Hrr1, an RNAi factor, also associates with $d g$ and $d h$ ncRNAs but not with $s n R 30$ or $a c t 1^{+}$RNA (Supplemental Fig. S1A). The association of Seb1 with $d g$ and $d h$ transcripts is maintained in a clr4D mutant that lacks $\mathrm{H} 3 \mathrm{~K} 9 \mathrm{me}$, indicating that this conserved heterochromatic methyl mark is not required for the association (Supplemental Fig. S1B). This is in contrast to RNAi factors that require $\mathrm{H} 3 \mathrm{~K} 9 \mathrm{me}$ to display a RIP signal on the $d g$ and $d h$ transcripts (Rougemaille et al. 2012).

We next isolated a mutation in $\operatorname{seb} 1^{+}$that was defective in heterochromatic silencing. Because $s e b 1^{+}$, like $N R D 1$, is an essential gene (Mitsuzawa et al. 2003), we mutagenized a gene targeting construct for the endogenous seb $1^{+}$ locus (Supplemental Fig. S2) and transformed this library into a strain harboring an ectopic heterochromatic silenc-

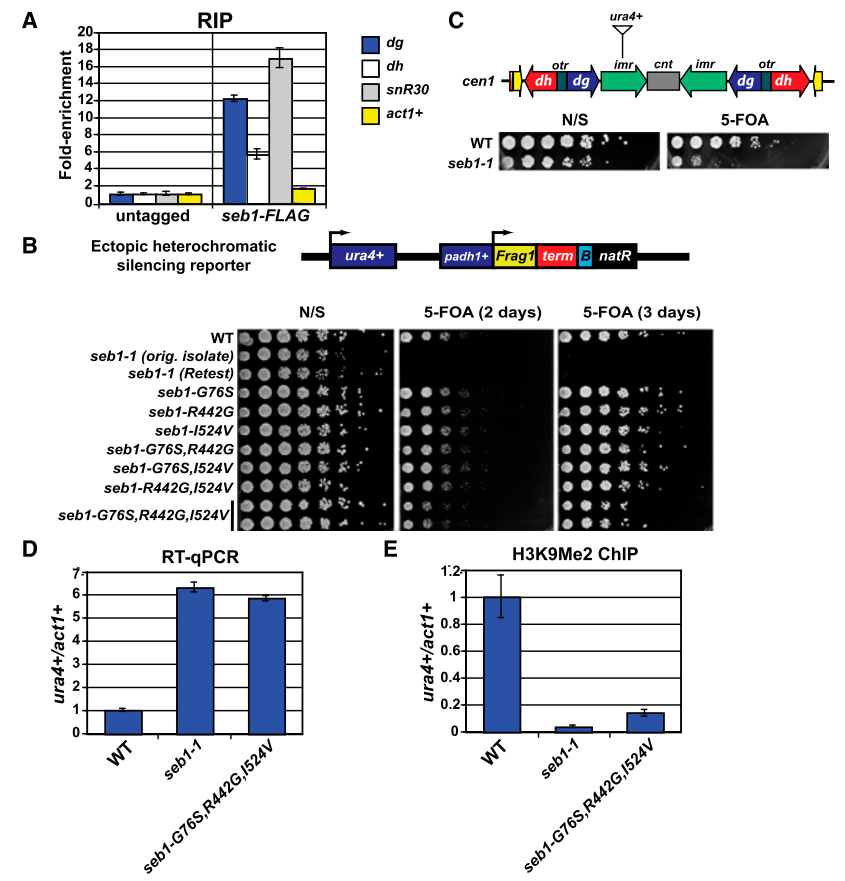

Figure 1. The seb1-1 mutant is defective in heterochromatic silencing at pericentromeric repeats. (A) RIP experiments measuring the enrichment of Sebl-Flag at $d g, d h$, and $a c t 1^{+}$transcripts and snR30 snoRNA in the wild-type strain. $(B$, top) Schematic of the ectopic heterochromatic silencing reporter construct used in the allele screen. (Bottom) Silencing assays of $s e b 1^{+}$mutations in the ectopic heterochromatic silencing reporter strain background. Cells were plated on nonselective rich YS medium (N/S) and YS medium with 5-FOA (5-FOA). (C, top) Schematic of centromere 1 with the ura ${ }^{+}$ reporter gene inserted in the innermost repeat (imr) region. (Bottom) Silencing assay of seb1-1 mutation in the pericentromeric ura $4^{+}$ reporter strain background. $(D, E)$ RT-qPCR analysis of $\mathrm{ura}^{+}$transcript levels (normalized to act $1^{+}$transcript levels) $(D)$ and ChIP analysis of $\mathrm{H} 3 \mathrm{~K} 9 \mathrm{me} 2$ levels at the $\mathrm{ura}^{+}$locus (normalized to $\mathrm{H} 3 \mathrm{~K} 9 \mathrm{me} 2$ levels at the $a c t 1^{+}$locus) (E) in the wild-type strain, the seb1-1 mutant, and the strain with three amino acid mutations in $s e b 1^{+}$. ing reporter system that we had developed for other studies. This system involves the insertion of a 2811base-pair (bp) fragment of $d h$ repeat downstream from the endogenous ura $4^{+}$gene such that the transcription of this fragment is driven by the $a d h 1^{+}$promoter (Supplemental Fig. S3A). This fragment ("fragment 1 ") was identified as a highly potent inducer of $\mathrm{ura}^{+}$silencing in a systematic study of the activities of $d g$ and $d h$ fragments (data not shown). Silencing by fragment 1 requires functional Clr4 and RNAi as well as the $a d h 1^{+}$promoter; the latter observation indicates that transcription of fragment 1 is required for silencing (Supplemental Fig. S3B). Silencing by fragment 1 also causes a decrease of $u r a 4^{+}$transcript level (Supplemental Fig. S3C) and an increase of H3K9me2 at the ura $^{+}$locus (Supplemental Fig. S3D). Screening of $\sim 10,000$ colonies produced by transformation of the $s e b 1^{+}$ mutant library yielded a single mutant that displays a defect in growth on 5-fluoroorotic acid (5-FOA) medium, which selects for strains with a silenced ura4 ${ }^{+}$gene (Supplemental Fig. S2). This allele, seb1-1, has seven

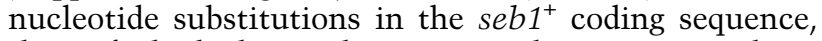
three of which change the amino acid sequence. Replacement of seb1 $1^{+}$with the seb1-1 allele in the parental strain recapitulates the 5-FOA phenotype (Fig. 1B). This mutation also causes a silencing defect at endogenous heterochromatin: A strain harboring a ura $4^{+}$reporter gene inserted into the innermost repeat (imr) region of centromere 1 displays reduced growth on 5-FOA when harboring the seb1-1 allele (Fig. 1C). In the ectopic heterochromatic silencing reporter strain, the seb1-1 mutation causes an accumulation of the $\mathrm{ura}^{+}$transcript (Fig. 1D) and a strong defect in $\mathrm{H} 3 \mathrm{~K} 9 \mathrm{me} 2$ at the $\mathrm{ura}_{4}^{+}$gene (Fig. 1E), supporting the growth defect observed using the 5-FOA assay.

We constructed all combinations of the three amino acid changes present in the seb1-1 allele (G76S, R442G, and $\mathrm{I524 \textrm {V } )}$ and used them to replace the wild-type seb1 $1^{+}$ sequence in the reporter strain. We found that all three mutations are required to produce a silencing defect on 5FOA medium (Fig. 1B). However, the mutant with the three amino acid changes (triple mutant) has a milder silencing defect on 5-FOA when compared with the original seb1-1 mutant. The silencing defect of the triple mutant is only obvious at $2 \mathrm{~d}$ of growth on 5-FOA, indicating that the silent mutations also contribute to the phenotype (Fig. 1B). Moreover, the triple mutant displays an intermediate defect in $\mathrm{H} 3 \mathrm{~K} 9 \mathrm{me} 2$ at the $\mathrm{ura}^{+}$locus when compared with the seb1-1 allele (Fig. 1E). The triple mutant displays accumulation of $\mathrm{ura}^{+}$transcript nearly comparable with that of the seb1-1 mutant (Fig. 1D), likely reflecting distinct sensitivities and thresholds to gene function of the 5-FOA, RNA, and H3K9me2 assays. As each of the four silent mutations (A45G, T132A, T1194C, and T1260A) changes the wild-type codon to a rarer synonymous codon (Forsburg 1994), they could impact protein expression. Indeed, the level of Seb1 protein is lower in the seb1-1 mutant when compared with its level in the wild-type seb1 $1^{+}$strain (Supplemental Fig. S4). Since the seb1-1 allele has a more robust silencing defect compared with the triple mutant, we used the seb1-1 allele in our further analyses.

We examined the effect of seb1-1 mutation on pericentromeric siRNA production using Northern hybridization and discovered that, unlike the clr $4 \Delta$ mutant, seb1-1 displays normal levels of pericentromeric siRNA accumulation (Fig. 2A). Consistent with the lack of a defect in 
A

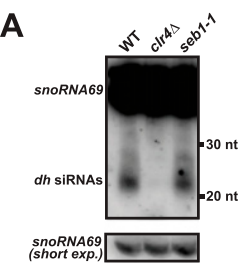

C

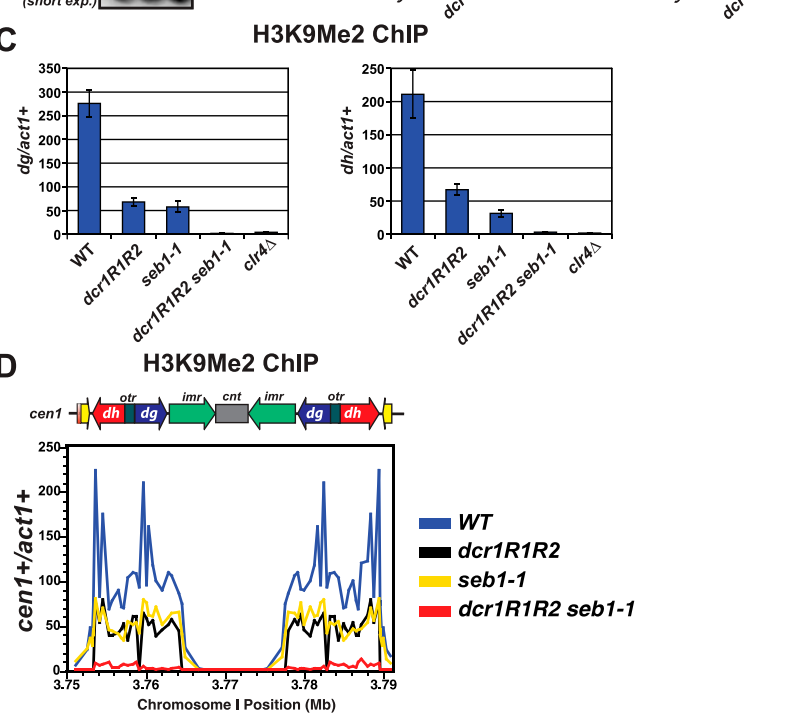

Figure 2. The seb1-1 mutation does not affect RNAi. $(A)$ Riboprobe siRNA Northern blot detecting $d h$ siRNAs in the wild-type, clr $4 \Delta$, and seb1-1 strains. snoRNA69 was used as a loading control. (B) RTqPCR analysis of $d g$ and $d h$ transcript levels (normalized to act $1^{+}$ transcript levels) in wild-type, seb1-1, and dcr1-R1R2 strains. $(C)$ ChIP analysis of $\mathrm{H} 3 \mathrm{~K} 9 \mathrm{me} 2$ levels at $d g$ and $d h$ repeats (normalized to $\mathrm{H} 3 \mathrm{~K} 9 \mathrm{me} 2$ levels at the act $1^{+}$locus) in a wild-type strain, seb1-1 and dcr1-R1R2 single mutants, the dcr1-R1R2 seb1-1 double mutant, and the clr $4 \Delta$ mutant. (D) ChIP analysis of H3K9me2 levels at centromere 1 (normalized to H3K9me2 levels at the act $1^{+}$locus) in wild-type strain, seb1-1 and $d c r 1-R 1 R 2$ single mutants, and the $d c r 1$ R1R2 seb1-1 double mutant.

siRNA production, the seb1-1 mutation does not cause an accumulation of $d g$ transcripts and only causes a slight increase in the level of $d h$ transcripts (Fig. 2B). In contrast, a catalytically dead Dcr1 mutant (dcr1-R1R2) (Colmenares et al. 2007) displays a dramatic increase in $d g$ and $d h$ transcript levels (Fig. 2B). We serendipitously discovered that seb1-1 confers a temperature-sensitive phenotype at $37^{\circ} \mathrm{C}$ (Supplemental Fig. S5A). As with the silencing phenotype, all seven mutations of $s e b 1^{+}$are required to produce the temperature sensitivity (Supplemental Fig. S5A). Northern hybridization demonstrated no defect in siRNA levels in the seb1-1 mutant, even at the nonpermissive temperature (Supplemental Fig. S5B). Taken together, these data argue that the silencing defect of the seb1-1 allele cannot simply be explained by a defect in RNAi.

To test whether Seb1 functions in the RNAi-independent pathway of heterochromatin formation, we measured H3K9me2 levels at the $d g$ and $d h$ repeats in the single and double combinations of seb1-1 and $d$ cr1-R1R2 alleles. Strikingly, while the levels of H3K9me2 are reduced by only threefold to fivefold in the single mutants, H3K9me2 is virtually abolished in the $d c r 1-R 1 R 2$ seb1-1 double mutant to background levels comparable with those measured in the clr4s mutant (Fig. 2C). We observed similar results across the entire pericentromeric region in chromatin immunoprecipitation and quantitative PCR (ChIP-qPCR) experiments using tiled pairs of primers (Fig. 2D; Braun et al. 2011). Moreover, we found that the seb1-1 mutation does not affect Chp1/RITS recruitment to $d g$ and $d h$ repeats (Supplemental Fig. S6). Together, these data demonstrate that Seb1 acts independently of RNAi to promote H3K9me at pericentromeric heterochromatin. To rule out the trivial possibility that the dcr1-R1R2 seb1-1 double mutation reduces the transcription of components of the CLRC, thereby affecting H3K9me2 indirectly, we performed RT-qPCR analyses and found no reduction in them in the mutant (Supplemental Fig. S7).

Although classical heterochromatin mediated by H3K9me was ancestrally lost during the evolution of $S$. cerevisiae, Nrdl has been reported to play a role in promoting a different type of silencing that occurs in the recombinant DNA (rDNA) repeats of budding yeast (Vasiljeva et al. 2008b). In this organism, cryptic unstable Pol II transcripts of unknown function are produced from rDNA, which is predominantly transcribed by Pol I. These Pol II transcripts are terminated and targeted for degradation by Nrd1 (Vasiljeva et al. 2008b). In Nrd1 mutants, the defect in termination and turnover leads to dramatic increases in the level of rDNA-derived Pol II transcripts and the accumulation of longer transcripts (Vasiljeva et al. 2008b). This transcriptional readthrough is associated with increased histone acetylation, reduced nucleosome occupancy, activation of inserted Pol II reporter genes, and increased recombination between rDNA repeats (Vasiljeva et al. 2008b). We tested whether Seb1 might act by such a mechanism in $S$. pombe pericentromeric regions by determining whether the seb1-1 mutation causes an increase in pericentromeric transcript size and levels in cells mutated for Dcr1. Northern hybridization using a riboprobe complementary to the region of the $d h$ repeats encoded by fragment 1 revealed heterogeneously sized transcripts produced from this region that accumulate in the $d c r 1-R 1 R 2$ mutant (Supplemental Fig. S8A). This pattern is consistent with previous reports (Zaratiegui et al. 2011). No obvious increase in transcript size was apparent, and there was no significant increase in transcript level in the seb1-1 dcr1-R1R2 double mutant relative to the $d c r 1-R 1 R 2$ single mutant (Supplemental Fig. S8A). Careful quantification using RT-qPCR also yielded no increase in the abundance of $d h$ transcript produced by the seb1-1 mutation in a $d c r 1-R 1 R 2$ genetic background (Supplemental Fig. S8B). These data indicate that mechanisms observed previously in $S$. cerevisiae cannot easily explain our observations in $S$. pombe.

Since it has been previously reported that the clr3s mutant, like the seb1-1 mutant, further reduces H3K9me2 in the dcr1s mutant (Yamada et al. 2005; Reyes-Turcu et al. 2011), we tested whether Seb1 and SHREC function in the same pathway. We quantitatively measured the levels of $\mathrm{H} 3 \mathrm{~K} 9 \mathrm{me} 2$ at $d g$ and $d h$ repeats in the $d c r 1-R 1 R 2$ seb1-1 and dcr1-R1R2 clr3s double mutants. Consistent with published results (Yamada et al. 2005; Reyes-Turcu et al. 2011), we observed that while H3K9me2 levels at the $d g$ and $d h$ repeats are modestly reduced in the $d c r 1-R 1 R 2$ single mutant, these levels are virtually eliminated in the dcr1-R1R2 clr3s double mutant, a phenotype strikingly similar to that of the $d c r 1-R 1 R 2$ seb1-1 double mutant (Fig. 3A). Furthermore, the elimination of H3K9me2 at $d g$ 


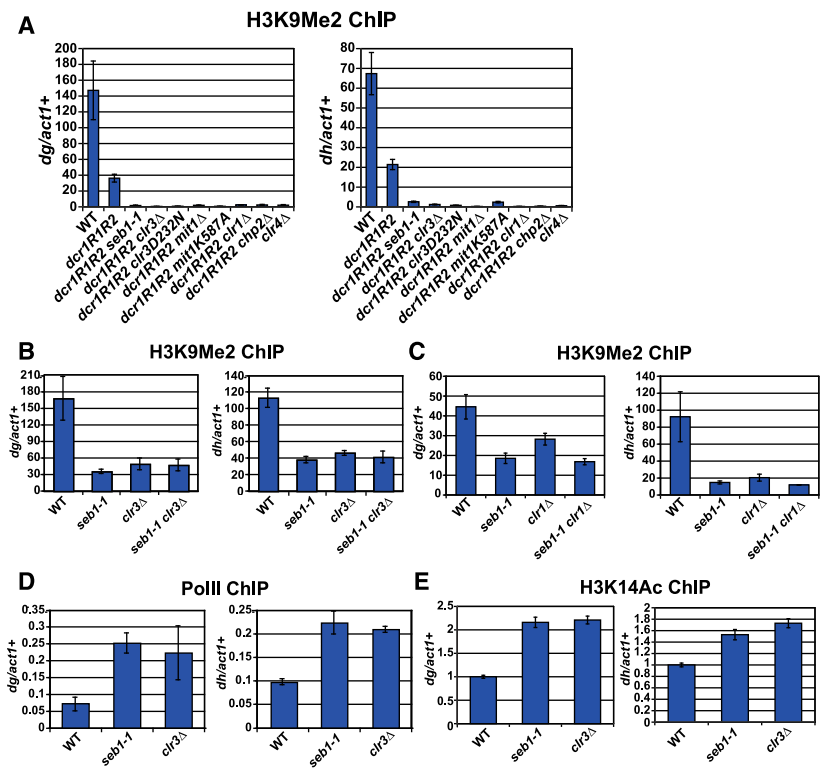

Figure 3. Seb1 functions in the same pathway as SHREC to promote H3K9me. (A) ChIP analysis of H3K9me2 levels at $d g$ and $d h$ repeats (normalized to $\mathrm{H} 3 \mathrm{~K} 9 \mathrm{me} 2$ levels at the act $1^{+}$locus) in wildtype, dcr1-R1R2, dcr1-R1R2 seb1-1, dcr1-R1R2 clr3A, dcr1-R1R2 clr3D232N, dcr1-R1R2 mit1s, dcr1-R1R2 mit1K587A, dcr1-R1R2

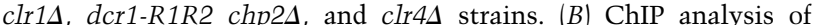
$\mathrm{H} 3 \mathrm{~K} 9 \mathrm{me} 2$ levels at $d g$ and $d \mathrm{~h}$ repeats (normalized to H3K9me2 levels at the act $1^{+}$locus) in the wild-type strain, the seb1-1 and clr3s single mutants, and the seb1-1 clr3s double mutant. (C) ChIP analysis of $\mathrm{H} 3 \mathrm{~K} 9 \mathrm{me} 2$ levels at $d g$ and $d h$ repeats (normalized to $\mathrm{H} 3 \mathrm{~K} 9 \mathrm{me} 2$ levels at the $a c t 1^{+}$locus) in the wild-type strain, the seb11 and clr1s single mutants, and the seb1-1 clr1s double mutant. $(D, E)$ ChIP analyses of Pol II $(D)$ and H3K14ac $(E)$ levels at $d g$ and $d h$ repeats (normalized to the levels at the $a c t 1^{+}$locus) in the wild-type strain and seb1-1 and clr3s single mutants.

and $d h$ repeats was also observed in strains that have the dcr1-R1R2 mutation combined with a deletion mutation that eliminates the Clr1, Chp2, or Mit1 subunits of SHREC (Fig. 3A). Finally, a catalytically dead point mutation in the Clr3 HDAC or the Mit1 ATPase domain also eliminates H3K9me in dcr1-R1R2 cells (Fig. 3A). These data demonstrate that all known activities of SHREC are required for the RNAi-independent pathway that promotes H3K9me at pericentromeric repeats.

To further test whether Seb1 functions in the same pathway as SHREC in promoting $\mathrm{H} 3 \mathrm{~K} 9 \mathrm{me}$, we compared H3K9me2 levels at $d g$ and $d h$ repeats of the seb1-1 clr3s double mutant with those of the corresponding single mutants. Significantly, the double and single mutants display very similar levels of H3K9me2, 20\%-30\% of wild-type levels (Fig. 3B). Similar results were obtained when comparing H3K9me2 levels at $d g$ and $d h$ repeats of the seb1-1 clr1s double mutant with the levels of the corresponding single mutants (Fig. 3C). These data provide strong genetic support that Seb1 and SHREC proteins function in the same pathway in promoting H3K9me at pericentromeric repeats. Moreover, we found that the seb1-1 mutation increases Pol II occupancy and H3K14 acetylation levels at $d g$ and $d h$ repeats (Figs. 3D,E), similar to that reported previously for the clr3s mutation (Sugiyama et al. 2007; Motamedi et al. 2008). To rule out the possibility that seb1-1 mutation indirectly affects heterochromatin assembly by decreasing the levels of
mRNAs that encode the subunits of SHREC, we measured their levels in the wild-type and seb1-1 strains. We found that the seb1-1 mutation does not reduce the levels of the mRNAs encoding any of the subunits of SHREC (Supplemental Fig. S9), further arguing for a direct role of Seb1 in heterochromatin formation.

Because Seb1 and SHREC function genetically in the same pathway, we hypothesized that Sebl physically interacts with SHREC to recruit it to pericentromeric repeats. An observation that supports this hypothesis is that the purification of the Clr2 subunit of SHREC was reported to have yielded two peptides from Seb1 (Supplemental Table S1; Motamedi et al. 2008). However, Seb1 peptide coverage was low and was not obtained in other purifications reported. To test for a physical interaction between Seb1 and SHREC using a more sensitive assay, we performed coimmunoprecipitation (co-IP) immunoblotting experiments. We found that Flag-tagged Seb1 coimmunoprecipitated with Clr3-myc (Fig. 4A) but not with a control protein (Cdc2 detected by anti-PSTAIRE), indicating that these two proteins physically interact in vivo. This interaction is not bridged by RNA, since we could still detect the interaction in the presence of RNase A (Fig. 4B). Seb1 also coimmunoprecipitated with other components of SHREC, including Clr1 and Mit1 (Fig. $4 \mathrm{C}, \mathrm{D})$. These data provide strong evidence that Seb1 and SHREC not only function in the same pathway, but also physically interact in vivo.

To gain further insight into how Seb1 functions to promote $\mathrm{H} 3 \mathrm{~K} 9 \mathrm{me}$, we tested whether Seb1 acts upstream of SHREC, as might be expected for a recruitment factor. We performed ChIP experiments to measure the enrichment of SHREC in strains harboring an epitope-tagged version of the proteins. We found that every subunit of SHREC tested (Clr3, Clr1, Mit1, and Chp2) is enriched at

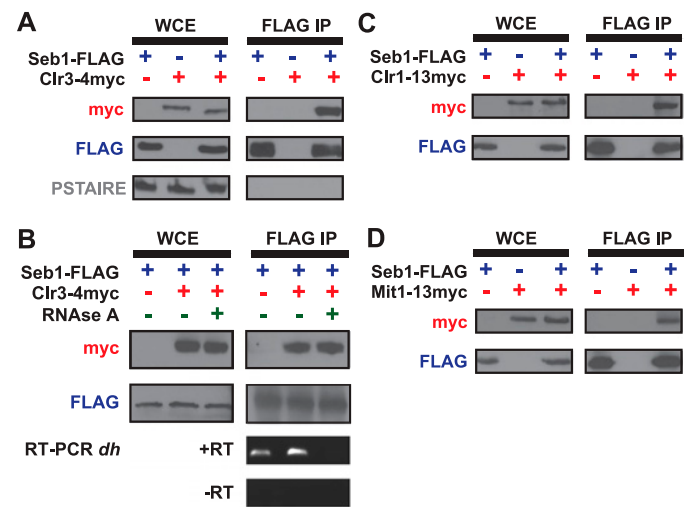

Figure 4. Sebl physically associates with SHREC in vivo. (A) Co-IP of Seb1 with Clr3. Strains expressing endogenously tagged Seb1CBP-2XFlag, Clr3-4myc, or both were subjected to anti-Flag immunoprecipitation. The whole-cell extract (WCE) and immunoprecipitated (IP) samples were detected by anti-myc (top), anti-Flag (middle), and anti-PSTAIRE (bottom) immunoblots; the latter serves as a loading and specificity control. (B) Co-IP of Seb1 with Clr3 in the presence of RNase A. The two bottom panels are images of agarose gels of RT-qPCR experiments to detect $d h$ transcripts in the presence $(+\mathrm{RT})$ or absence $(-\mathrm{RT})$ of reverse transcriptase. $(C, D)$ Co-IP of Seb1 with Clr1 and Mit1. Strains expressing endogenous levels of Seb1-CBP-2XFlag, Clr1-13myc, or both $(C)$ and Seb1-CBP-2XFlag, Mit1-13myc, or both $(D)$ were subjected to anti-Flag immunoprecipitation. The whole-cell extract (WCE) and immunoprecipitated (IP) samples were detected by anti-myc (top) and anti-Flag (bottom) immunoblots. 
both $d g$ and $d h$ repeats. However, in the seb1-1 mutant, this enrichment is abolished or strongly reduced (Figs. 5A-D). In contrast, RIP experiments demonstrate that Sebl still associates with $d g$ and $d h$ transcripts in cells lacking SHREC, indicating that Seb1 recruitment to these ncRNAs is not downstream from SHREC (Fig. 5E). Interestingly, we observed a reproducible increase in Seb1 association with $d g$ and $d h$ ncRNAs in the clr3s mutant but not the clr $4 \Delta$ mutant, suggesting negative regulation of Seb1 recruitment by Clr3 (Fig. 5E; Supplemental Fig. S1B). Together, these data support our hypothesis that Seb1 acts by recruiting SHREC to pericentromeric heterochromatin.

In $S$. pombe, heterochromatin is also found at the silent mating type locus and at subtelomeric regions. These loci contain DNA sequences that are similar to the pericentromeric $d g$ and $d h$ repeats. While Sebl is also associated with the $d g / d h$-like transcripts originating from these nonpericentromeric heterochromatic loci (Supplemental Fig. S10), the seb1-1 mutation does not affect SHREC recruitment to these loci (Supplemental Fig. S11). This is not completely unexpected, since there exists additional mechanisms of SHREC recruitment to the silent mating type locus and subtelomeric regions by DNA-binding proteins Atf1/Pcr1 and Taz1, respectively (Yamada et al. 2005; Sugiyama et al. 2007).

Our studies demonstrate an essential role for the ncRNA-binding protein Sebl in RNAi-independent pericentromeric heterochromatin formation through

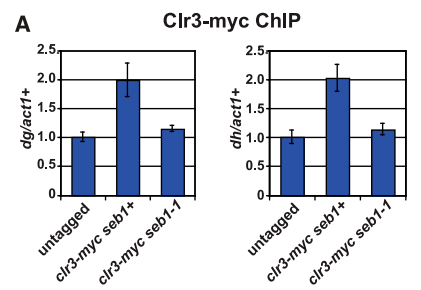

C
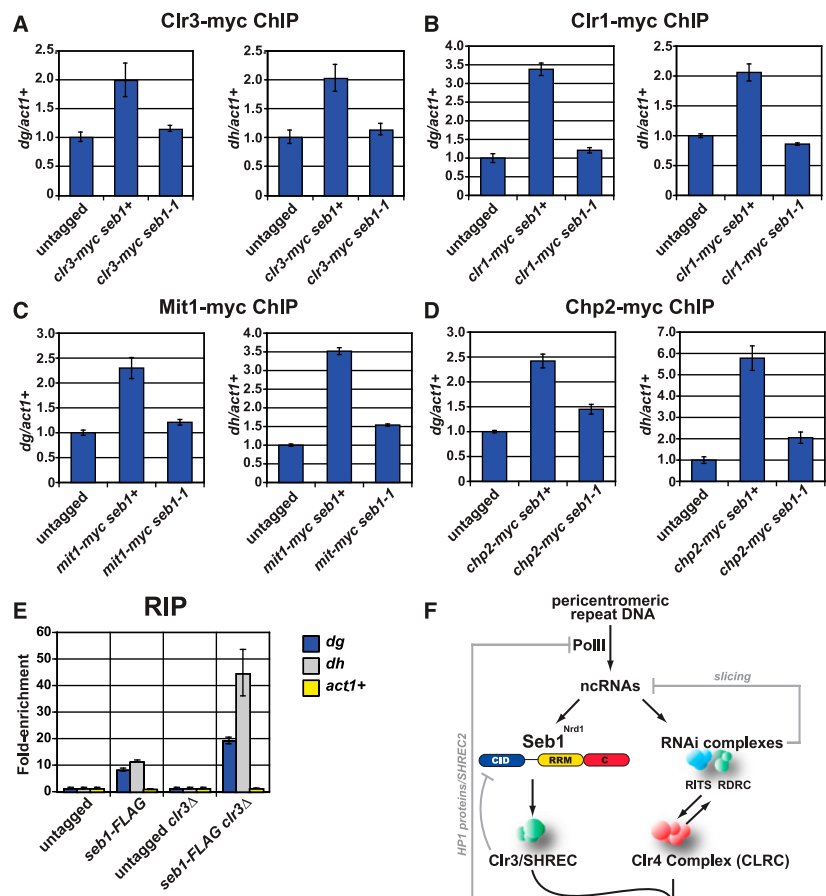

D
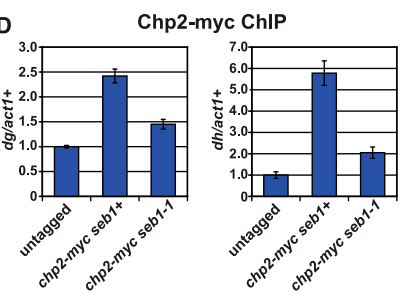

$\mathbf{F}$

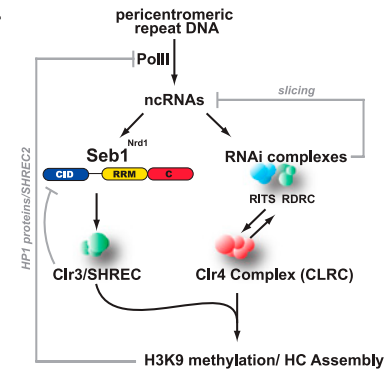

Figure 5. Seb1 recruits SHREC to pericentromeric heterochromatin. $(A-D)$ ChIP analyses of Clr3-myc, Clr1-myc, Mit1-myc, and Chp2-myc levels at $d g$ and $d h$ repeats (normalized to their levels at the $a c t 1^{+}$locus) in the wild-type and seb1-1 strains. (E) RIP experiments measuring the enrichment of Seb1-Flag at $d g$, $d h$, and act $1^{+}$transcripts in the wild-type and clr3s strains. $(F)$ Model for the RNAi-independent role of Seb1 in recruiting SHREC to pericentromeric heterochromatin.

recruitment of the chromatin-modifying complex SHREC. (Fig. 5F). SHREC may function to promote H3K9me by deacetylating the $\mathrm{H} 3$ tail, which is presumably necessary for its methylation by the CLRC. Alternatively, SHREC may have nonhistone substrates whose deacetylation promotes $\mathrm{H} 3 \mathrm{~K} 9 \mathrm{me}$. As HP1 proteins also promote the recruitment of SHREC, heterochromatin spread may be promoted by a positive feedback loop involving alternating cycles of histone deacetylation and methylation (Fig. 5F). Consequently, SHREC may be considered both an effector and a trigger of histone methylation.

Candidate recruiters of RNAi to heterochromatic regions in $S$. pombe include Pol II and the spliceosome (Djupedal et al. 2005; Kato et al. 2005; Bayne et al. 2008), which are essential for viability and operate at a very large number of genomic sites. Presumably, additional factors and signals determine where RNAi-related enzymes are recruited by these multiprotein complexes. Likewise, Seb1 also displays a strong association with nonheterochromatic snR30 snoRNA (Fig. 1A). Interestingly, however, we did not detect recruitment of Clr3 to the snR30 gene (Supplemental Fig. S12), suggesting that Seb1 may play SHREC-independent roles outside of heterochromatin. Our ongoing studies are aimed at defining the molecular cues (including potential RNA ligands) that distinguish Sebl's functions in heterochromatin versus euchromatin.

\section{Materials and methods}

\section{Strain construction and growth conditions}

Strains were constructed and grown by standard fission yeast methods as described previously (Rougemaille et al. 2012), except that 2 g/L 5-FOA was used in silencing assays.

\section{Immunoprecipitation experiments and RNA analyses}

ChIP, RIP, co-IP, and RT-qPCR experiments and Northern analyses were performed using standard molecular biology techniques as described in detail in the Supplemental Material.

\section{Acknowledgments}

We thank Danesh Moazed and Karl Ekwall for strains. We are grateful to Ratika Krishnamurty for sharing results of her experiments that were insightful during the revision process. We thank Jeff Corden, Sigurd Braun, Geeta Narlikar, Kristin Patrick, and members of the Madhani laboratory for critical comments on the manuscript. This work was supported by a grant from the National Institutes of Health (R01GM071801). D.B.M. was an American Heart Association predoctoral fellow. S.S. was a postdoctoral fellow of the Leukemia and Lymphoma Society.

\section{References}

Arigo JT, Eyler DE, Carroll KL, Corden JL. 2006. Termination of cryptic unstable transcripts is directed by yeast RNA-binding proteins Nrd1 and Nab3. Mol Cell 23: 841-851.

Bannister AJ, Zegerman P, Partridge JF, Miska EA, Thomas JO, Allshire RC, Kouzarides T. 2001. Selective recognition of methylated lysine 9 on histone $\mathrm{H} 3$ by the HP1 chromo domain. Nature 410: 120-124.

Bayne EH, Portoso M, Kagansky A, Kos-Braun IC, Urano T, Ekwall K, Alves F, Rappsilber J, Allshire RC. 2008. Splicing factors facilitate RNAi-directed silencing in fission yeast. Science 322: 602-606.

Braun S, Garcia JF, Rowley M, Rougemaille M, Shankar S, Madhani HD. 2011. The Cul4-Ddbl(Cdt)(2) ubiquitin ligase inhibits invasion of a boundaryassociated antisilencing factor into heterochromatin. Cell 144: 41-54.

Carroll KL, Pradhan DA, Granek JA, Clarke ND, Corden JL. 2004. Identification of cis elements directing termination of yeast nonpolyadenylated snoRNA transcripts. Mol Cell Biol 24: 6241-6252. 


\section{Marina et al.}

Colmenares SU, Buker SM, Buhler M, Dlakic M, Moazed D. 2007. Coupling of double-stranded RNA synthesis and siRNA generation in fission yeast RNAi. Mol Cell 27: 449-461.

Conrad NK, Wilson SM, Steinmetz EJ, Patturajan M, Brow DA, Swanson MS, Corden JL. 2000. A yeast heterogeneous nuclear ribonucleoprotein complex associated with RNA polymerase II. Genetics 154: 557-571.

Djupedal I, Portoso M, Spahr H, Bonilla C, Gustafsson CM, Allshire RC, Ekwall K. 2005. RNA Pol II subunit Rpb7 promotes centromeric transcription and RNAi-directed chromatin silencing. Genes Dev 19: 2301-2306.

Fischer T, Cui B, Dhakshnamoorthy J, Zhou M, Rubin C, Zofall M, Veenstra TD, Grewal SI. 2009. Diverse roles of HP1 proteins in heterochromatin assembly and functions in fission yeast. Proc Natl Acad Sci 106: 8998-9003.

Forsburg SL. 1994. Codon usage table for Schizosaccharomyces pombe. Yeast 10: 1045-1047.

Guttman M, Rinn JL. 2012. Modular regulatory principles of large noncoding RNAs. Nature 482: 339-346.

Guttman M, Amit I, Garber M, French C, Lin MF, Feldser D, Huarte M Zuk O, Carey BW, Cassady JP, et al. 2009. Chromatin signature reveals over a thousand highly conserved large non-coding RNAs in mammals. Nature 458: 223-227.

Kato H, Goto DB, Martienssen RA, Urano T, Furukawa K, Murakami Y. 2005. RNA polymerase II is required for RNAi-dependent heterochromatin assembly. Science 309: 467-469.

Khalil AM, Guttman M, Huarte M, Garber M, Raj A, Rivea Morales D, Thomas K, Presser A, Bernstein BE, van Oudenaarden A, et al. 2009. Many human large intergenic noncoding RNAs associate with chromatinmodifying complexes and affect gene expression. Proc Natl Acad Sci 106: 11667-11672.

Kim M, Vasiljeva L, Rando OJ, Zhelkovsky A, Moore C, Buratowski S. 2006. Distinct pathways for snoRNA and mRNA termination. Mol Cell 24: 723-734.

Lejeune E, Allshire RC. 2011. Common ground: Small RNA programming and chromatin modifications. Curr Opin Cell Biol 23: 258-265.

Meinhart A, Cramer P. 2004. Recognition of RNA polymerase II carboxyterminal domain by 3'-RNA-processing factors. Nature 430: 223-226.

Mitsuzawa H, Kanda E, Ishihama A. 2003. Rpb7 subunit of RNA polymerase II interacts with an RNA-binding protein involved in processing of transcripts. Nucleic Acids Res 31: 4696-4701.

Motamedi MR, Hong EJ, Li X, Gerber S, Denison C, Gygi S, Moazed D. 2008. HP1 proteins form distinct complexes and mediate heterochromatic gene silencing by nonoverlapping mechanisms. Mol Cell 32: $778-790$.

Nakayama J, Rice JC, Strahl BD, Allis CD, Grewal SI. 2001. Role of histone $\mathrm{H} 3$ lysine 9 methylation in epigenetic control of heterochromatin assembly. Science 292: 110-113.

Reyes-Turcu FE, Zhang K, Zofall M, Chen E, Grewal SI. 2011. Defects in RNA quality control factors reveal RNAi-independent nucleation of heterochromatin. Nat Struct Mol Biol 18: 1132-1138.

Rougemaille M, Braun S, Coyle S, Dumesic PA, Garcia JF, Isaac RS, Libri D, Narlikar GJ, Madhani HD. 2012. Ers1 links HP1 to RNAi. Proc Natl Acad Sci 109: 11258-11263.

Sadaie M, Kawaguchi R, Ohtani Y, Arisaka F, Tanaka K, Shirahige K, Nakayama J. 2008. Balance between distinct HP1 family proteins controls heterochromatin assembly in fission yeast. Mol Cell Biol 28: 6973-6988.

Steinmetz EJ, Brow DA. 1996. Repression of gene expression by an exogenous sequence element acting in concert with a heterogeneous nuclear ribonucleoprotein-like protein, Nrd1, and the putative helicase Sen1. Mol Cell Biol 16: 6993-7003.

Steinmetz EJ, Brow DA. 1998. Control of pre-mRNA accumulation by the essential yeast protein Nrd1 requires high-affinity transcript binding and a domain implicated in RNA polymerase II association. Proc Nat1 Acad Sci 95: 6699-6704.

Steinmetz EJ, Conrad NK, Brow DA, Corden JL. 2001. RNA-binding protein Nrd1 directs poly(A)-independent 3 '-end formation of RNA polymerase II transcripts. Nature 413: 327-331.

Sugiyama T, Cam HP, Sugiyama R, Noma K, Zofall M, Kobayashi R, Grewal SI. 2007. SHREC, an effector complex for heterochromatic transcriptional silencing. Cell 128: 491-504.

Thiebaut M, Kisseleva-Romanova E, Rougemaille M, Boulay J, Libri D. 2006. Transcription termination and nuclear degradation of cryptic unstable transcripts: A role for the nrd1-nab3 pathway in genome surveillance. Mol Cell 23: 853-864.

Thon G, Verhein-Hansen J. 2000. Four chromo-domain proteins of Schizosaccharomyces pombe differentially repress transcription at various chromosomal locations. Genetics 155: 551-568.

Vasiljeva L, Kim M, Mutschler H, Buratowski S, Meinhart A. 2008a. The Nrd1-Nab3-Sen1 termination complex interacts with the Ser5phosphorylated RNA polymerase II C-terminal domain. Nat Struct Mol Biol 15: 795-804.

Vasiljeva L, Kim M, Terzi N, Soares LM, Buratowski S. 2008b. Transcription termination and RNA degradation contribute to silencing of RNA polymerase II transcription within heterochromatin. Mol Cell 29: 313-323.

Verdel A, Jia S, Gerber S, Sugiyama T, Gygi S, Grewal SI, Moazed D. 2004. RNAi-mediated targeting of heterochromatin by the RITS complex. Science 303: 672-676.

Verdel A, Vavasseur A, Le Gorrec M, Touat-Todeschini L. 2009. Common themes in siRNA-mediated epigenetic silencing pathways. Int $J$ DeV Biol 53: 245-257.

Yamada T, Fischle W, Sugiyama T, Allis CD, Grewal SI. 2005. The nucleation and maintenance of heterochromatin by a histone deacetylase in fission yeast. Mol Cell 20: 173-185.

Zaratiegui M, Castel SE, Irvine DV, Kloc A, Ren J, Li F, de Castro E, Marin L, Chang AY, Goto D, et al. 2011. RNAi promotes heterochromatic silencing through replication-coupled release of RNA Pol II. Nature 479: $135-138$

Zhang K, Mosch K, Fischle W, Grewal SI. 2008. Roles of the Clr4 methyltransferase complex in nucleation, spreading and maintenance of heterochromatin. Nat Struct Mol Biol 15: 381-388. 


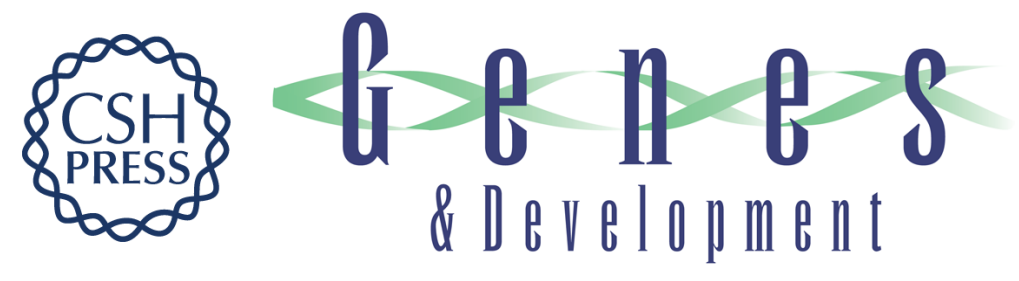

\section{A conserved ncRNA-binding protein recruits silencing factors to heterochromatin through an RNAi-independent mechanism}

Diana B. Marina, Smita Shankar, Prashanthi Natarajan, et al.

Genes Dev. 2013, 27:

Access the most recent version at doi:10.1101/gad.226019.113

\section{Supplemental http://genesdev.cshlp.org/content/suppl/2013/09/06/27.17.1851.DC1 \\ Material}

References This article cites 36 articles, 14 of which can be accessed free at:

http://genesdev.cshlp.org/content/27/17/1851.full.html\#ref-list-1

Creative This article is distributed exclusively by Cold Spring Harbor Laboratory Press for the first

Commons six months after the full-issue publication date (see

License http://genesdev.cshlp.org/site/misc/terms.xhtml). After six months, it is available under a Creative Commons License (Attribution-NonCommercial 3.0 Unported), as described at http://creativecommons.org/licenses/by-nc/3.0/.

Email Alerting Receive free email alerts when new articles cite this article - sign up in the box at the top Service right corner of the article or click here.

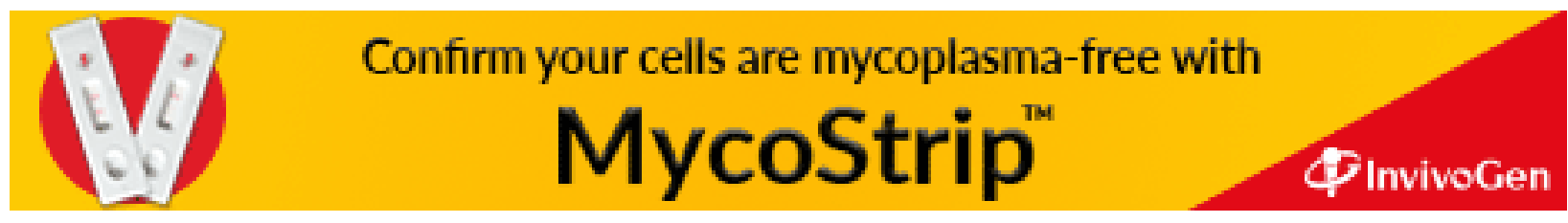

